九州大学学術情報リポジトリ

Kyushu University Institutional Repository

\title{
RATES OF CONVERGENCE IN DISTRIBUTION OF A LINEAR COMBINATION OF U-STATISTICS FOR NON- DEGENERATE KERNEL
}

Toda, Koichiro

Graduate School of Science and engineering, Kagoshima University

Yamato, Hajime

Department of Mathematics and Computer Science, Kagoshima University

https://doi.org/10.5109/13514

出版情報: Bulletin of informatics and cybernetics. 34 (2)，pp.133-141，2002-12. Research Association of Statistical Sciences

バージョン :

権利関係 : 


\title{
RATES OF CONVERGENCE IN DISTRIBUTION OF A LINEAR COMBINATION OF U-STATISTICS FOR NON-DEGENERATE KERNEL
}

By

\author{
Koichiro TODA* and Hajime Yamato ${ }^{\dagger}$
}

\begin{abstract}
As an estimator of an estimable parameter, we consider a linear combination of U-statistics introduced by Toda and Yamato (2001). As a special case, this statistic includes the V-statistic and LB-statistic. In case that the kernel is not degenerate, this linear combination of U-statistics converges to normal distribution. We show some rates of convergence different from Berry-Esseen bound.
\end{abstract}

Key Words and Phrases: Estimable parameter, Rate of convergence, linear combination of U-statistics, V-statistics.

\section{Introduction}

Let $\theta(F)$ be an estimable parameter of an unknown distribution $F$ and $g\left(x_{1}, \ldots, x_{k}\right)$ be its kernel of degree $k(\geq 2)$. We assume that the kernel $g$ is symmetric and not degenerate. Let $X_{1}, \ldots, X_{n}$ be a random sample of size $n$ from the distribution $F$.

As an estimator of $\theta(F)$, Toda and Yamato (2001) introduces a linear combination $Y_{n}$ of U-statistics as follows: Let $w\left(r_{1}, \ldots, r_{j} ; k\right)$ be a nonnegative and symmetric function of positive integers $r_{1}, \ldots, r_{j}$ such that $r_{1}+\cdots+r_{j}=k$ for $j=1, \ldots, k$. We assume that at least one of $w\left(r_{1}, \ldots, r_{j} ; k\right)$ 's is positive. For $j=1, \ldots, k$, let $g_{(j)}\left(x_{1}, \ldots, x_{j}\right)$ be the kernel given by

$$
g_{(j)}\left(x_{1}, \ldots, x_{j}\right)=\frac{1}{d(k, j)} \sum_{r_{1}+\cdots+r_{j}=k}^{+} w\left(r_{1}, \ldots, r_{j} ; k\right) g(\underbrace{x_{1}, \ldots, x_{1}}_{r_{1}}, \ldots, \underbrace{x_{j}, \ldots, x_{j}}_{r_{j}}),
$$

where the summation $\sum_{r_{1}+\cdots+r_{j}=k}^{+}$is taken over all positive integers $r_{1}, \ldots, r_{j}$ satisfying $r_{1}+\cdots+r_{j}=k$ with $j$ and $k$ fixed and $d(k, j)=\sum_{r_{1}+\cdots+r_{j}=k}^{+} w\left(r_{1}, \ldots, r_{j} ; k\right)$ for $j=1,2, \ldots, k$. Let $U_{n}^{(j)}$ be the U-statistic associated with kernel $g_{(j)}\left(x_{1}, \ldots, x_{j} ; k\right)$ for $j=1, \ldots, k$. The kernel $g_{(j)}\left(x_{1}, \ldots, x_{j} ; k\right)$ is symmetric because of the symmetry of $w\left(r_{1}, \ldots, r_{j} ; k\right)$. If $d(k, j)$ is equal to zero for some $j$, then the associated $w\left(r_{1}, \ldots, r_{j} ; k\right)$ 's are equal to zero. In this case, we let the corresponding statistic $U_{n}^{(j)}$ be zero. Note that

* Graduate School of Science and Engineering, Kagoshima University, Kagoshima 890-0065, Japan.

$\dagger$ Department of Mathemstics and Computer Science, Kagoshima University, Kagoshima 890-0065, Japan. 
$U_{n}^{(k)}=U_{n}$ for $w(1, \ldots, 1 ; k)>0$, because of $g_{(k)}=g$. The statistics $Y_{n}$ is given by

$$
Y_{n}=\frac{1}{D(n, k)} \sum_{j=1}^{k} d(k, j)\left(\begin{array}{l}
n \\
j
\end{array}\right) U_{n}^{(j)}
$$

where $D(n, k)=\sum_{j=1}^{k} d(k, j)\left(\begin{array}{l}n \\ j\end{array}\right)$. Since w's are nonnegative and at least one of them is pasitive, $D(n, k)$ is positive. $Y_{n}$ includes important statistjes as shown in the following examples.

EXAMPLE 1. Let $w$ be the function given by $w(1,1, \ldots, 1 ; k)=1$ and $w\left(r_{1}, \ldots, r_{j} ; k\right)=$ 0 for positive integers $r_{1}, \ldots, r_{j}$ such that $r_{1}+\cdots+r_{j}=k$ for $j=1, \ldots, k-1$. Then the corresponding statistic $Y_{n}$ is equal to U-statistic $U_{n}$, which is given by

$$
U_{n}=\left(\begin{array}{l}
n \\
k
\end{array}\right)^{-1} \sum_{1 \leq j_{1}<\cdots<j_{k} \leq n} g\left(X_{j_{1}}, \ldots, X_{j_{k}}\right),
$$

where $\sum_{1 \leq j_{1}<\cdots<j_{k} \leq n}$ denotes the summation over all integers $j_{1}, \ldots, j_{k}$ satisfying $1 \leq$ $j_{1}<\cdots<j_{k} \leq n$.

EXAMPLE 2. Let $w$ be the function given by $w\left(r_{1}, \ldots, r_{j} ; k\right)=1$ for positive integers $r_{1}, \ldots, r_{j}$ such that $r_{1}+\cdots+r_{j}=k$ for $j=1, \ldots, k$. Then the corresponding statistic $Y_{n}$ is equal to the LB-statistic $B_{n}$ given by

$$
B_{n}=\left(\begin{array}{c}
n+k-1 \\
k
\end{array}\right)^{-1} \sum_{r_{1}+\ldots+r_{n}=k} g(\underbrace{X_{1}, \ldots, X_{1}}_{r_{1}}, \ldots, \underbrace{X_{n}, \ldots, X_{n}}_{r_{n}}),
$$

where $\sum_{r_{1}+\cdots+r_{n}=k}$ denotes the summation over all non-negative integers $r_{1}, \ldots, r_{n}$ satisfying $r_{1}+\cdots+r_{n}=k$.

EXAMPLE 3. Let $w$ be the function given by $w\left(r_{1}, \ldots, r_{j} ; k\right)=k ! /\left(r_{1} ! \cdots r_{j} !\right)$ for positive integers $r_{1}, \ldots, r_{j}$ such that $r_{1}+\cdots+r_{j}=k$ for $j=1, \ldots, k$. Then the corresponding statistic $Y_{n}$ is equal to the $V$-statistic $V_{n}$ given by

$$
V_{n}=\frac{1}{n^{k}} \sum_{j_{1}=1}^{n} \cdots \sum_{j_{k}=1}^{n} g\left(X_{j_{1}}, \ldots, X_{j_{k}}\right)
$$

(See Toda and Yamato, 2001).

EXAMPLE 4. Let $w$ be the function given by $w\left(r_{1}, \ldots, r_{j} ; k\right)=k ! /\left(r_{1} \cdots r_{j}\right)$ for positive integers $r_{1}, \ldots, r_{j}$ such that $r_{1}+\cdots+r_{j}=k$ for $j=1, \ldots, k$. Then, for example, the corresponding statistic $Y_{n}$ for the third central moment of the distribution $F$ is given by

$$
S_{n}=\frac{n}{n^{2}+1} \sum_{i=1}^{n}\left(X_{i}-\bar{X}\right)^{3},
$$

where $\bar{X}$ is the sample mean of $X_{1}, \ldots, X_{n}$ (see Nomachi et al., 2002). 
For the non-degenerate kernel $g$, U-statistic $U_{n}$ converges to normal distribution. The purpose of this paper is to show some rates of convergences different from the Berry-Esseen bound, for linear combination of U-statistics $Y_{n}$ given by (1.3). In Section 2, we quote three rates of convergence different from the Berry-Esseen bound, from Zhao (1983), Zhao and Chen (1983), Koroljuk and Borovskich (1994) and Borovskikh (1996). Furthermore we give a new rate described by using a polynomial. In Section 3, for the statistic $Y_{n}$ we shall show three rates of convergence to normal distribution, using the propositions of Section 2. Furthermore, we give a rate different from these ones, using a polynomial.

\section{Rates of convergence for U-statistics}

For kernel $g\left(x_{1}, \ldots, x_{k}\right)$, we put

$$
\begin{gathered}
\psi_{1}\left(x_{1}\right)=E\left(g\left(X_{1}, \ldots, X_{k}\right) \mid X_{1}=x_{1}\right) \\
\psi_{2}\left(x_{1}, x_{2}\right)=E\left(g\left(X_{1}, \ldots, X_{k}\right) \mid X_{1}=x_{1}, X_{2}=x_{2}\right) \\
g^{(1)}\left(x_{1}\right)=\psi_{1}\left(x_{1}\right)-\theta, \quad \sigma_{1}^{2}=E\left[g^{(1)}\left(X_{1}\right)^{2}\right]>0
\end{gathered}
$$

and

$$
g^{(2)}\left(x_{1}, x_{2}\right)=\psi_{2}\left(x_{1}, x_{2}\right)-\psi_{1}\left(x_{1}\right)-\psi_{1}\left(x_{2}\right)-\theta .
$$

Let $\Phi(x)$ be the standard normal distribution function. We shall quote two rates of convergence of the distribution for U-statistic $U_{n}$.

LEMMA 2.1. (Koroljuk and Borovskich, 1994, Theorem 6.2.4) If for some $0 \leq \delta \leq 1$ kernel $g$ satisfies the conditions

$$
\sigma_{1}>0, \quad E\left|g^{(1)}\left(X_{1}\right)\right|^{2+\delta}<\infty, \quad E\left|g\left(X_{1}, \ldots, X_{k}\right)\right|^{\frac{4+\delta}{3}}<\infty,
$$

then

$$
\sup _{-\infty<x<\infty}\left|P\left(\frac{\sqrt{n}}{k \sigma_{1}}\left(U_{n}-\theta\right) \leq x\right)-\Phi(x)\right|=O\left(n^{-\frac{6}{2}}\right)
$$

as $n \rightarrow \infty$, and for $\delta=0$ we can replace $O(1)$ on the right-hand side by o(1).

LEMMA 2.2. (Koroljuk and Borovskich, 1994, Theorem 6.2.5, Zhao, 1983) Let $\sigma_{1}>$ 0 and $E\left|g\left(X_{1}, \ldots, X_{k}\right)\right|^{3}<\infty$. Then the inequality

$$
\left|P\left(\frac{\sqrt{n}}{k \sigma_{1}}\left(U_{n}-\theta\right) \leq x\right)-\Phi(x)\right| \leq \frac{C}{\sqrt{n}\left(1+x^{2}\right)}
$$

holds for all $x \in R$, where $C$ depends on kernel $g$ only via $\sigma_{1}$ and $E|g|^{3}$ and does not depend on $x$ and $n$.

Hereafter we use $C, C_{1}, C_{2}, C_{3}, \ldots$ as generic constants which do not depend on $x$ and $n$. We shall show the similar result to (2.2) for $Y$-statistic $Y_{n}$. For this purpose we quote the following. 
LEMMA 2.3. (Zhao, 1983, Lemma 7) Suppose that $W_{n}=W_{n 1}+W_{n 2}, n=1,2, \ldots$ be a sequence of random variables. Denote the distribution functions of $W_{n}$ and $W_{n 1}$ by $F_{n}$ and $F_{n 1}$, respectively. If

$$
\left|F_{n 1}-\Phi(x)\right| \leq \frac{C_{1}}{\sqrt{n}\left(1+x^{2}\right)}
$$

for all $x \in R$ and for $|x| \geq 1$

$$
P\left(\left|W_{n 2}\right| \geq \frac{C_{2}}{\sqrt{n}}|x|\right) \leq \frac{C_{3}}{\sqrt{n}\left(1+x^{2}\right)},
$$

then for all $x \in R$

$$
\left|F_{n}-\Phi(x)\right| \leq \frac{C_{4}}{\sqrt{n}\left(1+x^{2}\right)}
$$

In the following lemma, we consider kernel $g$ of degree $k=2$.

LEMMA 2.4. (Zhao and Chen, 1983) Let $\sigma_{1}>0$ and $E\left|g\left(X_{1}, X_{2}\right)\right|^{3}<\infty$. Then the inequality

$$
\left|P\left(\frac{\sqrt{n}}{2 \sigma_{1}}\left(U_{n}-\theta\right) \leq x\right)-\Phi(x)\right| \leq \frac{C}{\sqrt{n}(1+|x|)^{3}}
$$

holds for all $n(\geq 2)$ and all $x \in R$.

For this lemma, see also, Koroljuk and Borovskich (1994), Theorem 6.2.6 and Borovskikh (1996), Theorem 6.4.1. We shall show the similar result to (2.3) for the Y-statistic $Y_{n}$. For this purpose we quote the following.

LEMMA 2.5. (Zhao and Chen, 1983, Lemma 3) Suppose that $W_{n}=W_{n 1}+W_{n 2}$, $n=1,2, \ldots$ be a sequence of random variables. Denote the distribution functions of $W_{n}$ and $W_{n 1}$ by $F_{n}$ and $F_{n 1}$, respectively. If

$$
\left|F_{n 1}-\Phi(x)\right| \leq \frac{C_{1}}{\sqrt{n}(1+|x|)^{3}}
$$

for all $x \in R$ and for $|x| \geq 1$

$$
P\left(\left|W_{n 2}\right| \geq \frac{C_{2}}{\sqrt{n}}|x|\right) \leq \frac{C_{3}}{\sqrt{n}(1+|x|)^{3}},
$$

then for all $x \in R$

$$
\left|F_{n}-\Phi(x)\right| \leq \frac{C_{4}}{\sqrt{n}(1+|x|)^{3}} .
$$

Again we consider the kernel of degree $k \geq 2$. Let us consider a bound related with a polynomial including $1+x^{2}$ of $(2.2)$ and $(1+x)^{3}$ of $(2.3)$. If we allow $n$ to depend on $x$, then we have the following. 
THEOREM 2.6. Let $\sigma_{1}>0$ and $E\left|g\left(X_{1}, \ldots, X_{k}\right)\right|^{3}<\infty$. In addition, we suppose that $\lim _{|t| \rightarrow \infty}|\eta(t)|<1$. Let $p$ be a polynomial which is positive and increasing over $[0, \infty)$. Then inequality

$$
\left|P\left(\frac{\sqrt{n}}{k \sigma_{1}}\left(U_{n}-\theta\right) \leq x\right)-\Phi(x)\right| \leq \frac{C}{\sqrt{n} p(|x|)}, \quad x \in R
$$

holds for a sufficiently large $n$ which depends on $x$.

Before its proof we note the Berry-Esseen bound and the Edgeworth expansion. Let $\phi$ be the density of the standard normal distribution and

$$
\kappa_{3}=\sigma_{1}^{-3}\left[E\left[\left(g^{(1)}(X)\right)^{3}\right]+3(k-1) E\left[g^{(1)}\left(X_{1}\right) g^{(1)}\left(X_{2}\right) g^{(2)}\left(X_{1}, X_{2}\right)\right]\right] .
$$

Under the condition of this theorem we have the Berry-Esseen bound

$$
\sup _{-\infty<x<\infty}\left|P\left(\frac{\sqrt{n}}{k \sigma_{1}}\left(U_{n}-\theta\right) \leq x\right)-\Phi(x)\right| \leq \frac{C_{1}}{\sqrt{n}} .
$$

and the Edgeworth expansion

$$
\sup _{-\infty<x<\infty}\left|P\left(\frac{\sqrt{n}}{k \sigma_{1}}\left(U_{n}-\theta\right) \leq x\right)-Q_{n}(x)\right| \leq \frac{\epsilon_{n}}{\sqrt{n}},
$$

where

$$
Q_{n}(x)=\Phi(x)-\frac{1}{6 \sqrt{n}}\left(x^{2}-1\right) \kappa_{3} \phi(x)
$$

and $\varepsilon_{n} \rightarrow 0$ as $n \rightarrow \infty$ (see, for example, Maesono and Yamato, 1994).

Proof of Theorem 2.6. Let $M$ be a positive constant such that

$$
\left|x^{2}-1\right| p(|x|) \phi(x) \leq 1 \text { for }|x| \geq M .
$$

By the definition of $Q_{n}$ we have

$$
\begin{gathered}
I_{n}=\left|P\left(\frac{\sqrt{n}}{k \sigma_{1}}\left(U_{n}-\theta\right) \leq x\right)-\Phi(x)\right| \\
\leq \sup \left|P\left(\frac{\sqrt{n}}{k \sigma_{1}}\left(U_{n}-\theta\right) \leq x\right)-Q_{n}(x)\right|+\frac{1}{6 \sqrt{n}}\left|\left(x^{2}-1\right) \kappa_{3}\right| \phi(x) .
\end{gathered}
$$

For a given $x$, we can choose a sufficiently large $n$ such that $\epsilon_{n}<1 / p(|x|)$. Using (2.6), for $|x| \geq M$, we have for a sufficiently large $n$

$$
I_{n} \leq \frac{1}{\sqrt{n} p(|x|)}+\frac{\kappa_{3}}{6 \sqrt{n} p(|x|)}=\frac{C_{1}}{\sqrt{n} p(|x|)}
$$

If $|x| \leq M$, then $p(|x|)$ is bounded and $1 / p(M) \leq 1 / p(|x|) \leq 1 / p(0)$. Therefore by (2.5) we have

$$
I_{n} \leq \frac{C_{2}}{\sqrt{n} p(|x|)}
$$

Thus we get (2.4). 


\section{Rates of convergence for $\mathbf{Y}$-statistics}

If $d(k, k)=w(1, \ldots, 1 ; k)>0$, then there exists a constant $\beta(\geq 0)$ such that

$$
\frac{d(k, k)}{D(n, k)}\left(\begin{array}{l}
n \\
k
\end{array}\right)=1-\frac{\beta}{n}+O\left(\frac{1}{n^{2}}\right)
$$

and

$$
\sum_{j=1}^{k-1} \frac{d(k, j)}{D(n, k)}\left(\begin{array}{l}
n \\
j
\end{array}\right)=\frac{\beta}{n}+O\left(\frac{1}{n^{2}}\right) .
$$

For U-statistic $U_{n}, \beta=0$. In the following we assume that

$$
\beta>0
$$

because the corresponding results for U-statistic are given in Section 2. For V-statistic $V_{n}$ and S-statistic $S_{n}, \beta=k(k-1) / 2$. For the LB-statistic $B_{n}, \beta=k(k-1)$.

As stated in Toda and Yamato (2001), we can write

$$
Y_{n}=U_{n}+R_{n}
$$

and $R_{n}$ satisfies the following: For $r(\geq 1)$ and integers $j_{1}, \ldots, j_{k}\left(1 \leq j_{1} \leq \cdots \leq j_{k} \leq k\right)$, we assume $E\left|g\left(X_{j_{1}}, \ldots, X_{j_{k}}\right)\right|^{r}<\infty$. Then we have

$$
E\left|R_{n}-E R_{n}\right|^{r} \leq C_{1} n^{-\frac{3 r}{2}}, \quad r \geq 2
$$

and

$$
E\left|R_{n}-E R_{n}\right|^{r} \leq C_{2} n^{-(2 r-1)}, \quad 1 \leq r<2,
$$

(we note here these inequalities hold even if $r$ is not integer by the reason of the proof of Proposition 3.6 of Toda and Yamato, 2001). From (3.1), we have

$$
Y_{n}-E Y_{n}=U_{n}-\theta+\left(R_{n}-E R_{n}\right) \text {. }
$$

THEOREM 3.1. If for some $0 \leq \delta \leq 1$ the kernel $g$ satisfy the conditions

$$
\sigma_{1}>0, \quad E\left|g^{(1)}\left(X_{1}\right)\right|^{2+\delta}<\infty, \quad E\left|g\left(X_{1}, \ldots, X_{k}\right)\right|^{\frac{4+\delta}{3}}<\infty,
$$

and

$$
E\left|g\left(X_{j_{1}}, \ldots, X_{j_{k}}\right)\right|^{\frac{s+s}{\delta}}<\infty, \quad 1 \leq j_{1} \leq \cdots \leq j_{k} \leq k
$$

then

$$
\sup _{-\infty<x<\infty}\left|P\left(\frac{\sqrt{n}}{k \sigma_{1}}\left(Y_{n}-E Y_{n}\right) \leq x\right)-\Phi(x)\right|=O\left(n^{-\frac{5}{2}}\right)
$$

as $n \rightarrow \infty$.

Proof. Let $G_{n}$ and $\Phi_{n}$ be the distribution functions of $\left(\sqrt{n} /\left(k \sigma_{1}\right)\right)\left[Y_{n}-E Y_{n}\right]$ and $\left(\sqrt{n} /\left(k \sigma_{1}\right)\right)\left[U_{n}-\theta\right]$, respectively. Then for any $\varepsilon>0$

$$
\sup \left|G_{n}(x)-\Phi(x)\right| \leq \sup \left|\Phi_{n}(x)-\Phi(x)\right|+P\left(\frac{\sqrt{n}\left|R_{n}-E R_{n}\right|}{k \sigma_{1}} \geq \varepsilon\right)+\frac{\varepsilon}{\sqrt{2 \pi}}
$$


(see, for excample, Lee, 1990, p.187). By taking $\varepsilon=n^{-\delta / 2}$ and using Markov's inequality and (3.3),

$$
P\left(\frac{\sqrt{n}\left|R_{n}-E R_{n}\right|}{k \sigma_{1}} \geq \varepsilon\right) \leq \frac{1}{\varepsilon^{\frac{8+\delta}{\delta}}} E\left[\frac{\sqrt{n}\left|R_{n}-E R_{n}\right|}{k \sigma_{1}}\right]^{\frac{8 f \delta}{\delta}} \leq C n^{-\frac{\delta}{2}+\frac{1}{12}(\delta+12)(\delta-1)} .
$$

Since $0 \leq \delta \leq 1$,

$$
P\left(\frac{\sqrt{n}\left|R_{n}-E R_{n}\right|}{k \sigma_{1}} \geq \varepsilon\right)=O\left(n^{-\frac{5}{2}}\right) .
$$

Thus applying this relation and Lemma 2.1 to (3.4) with $\varepsilon=n^{-\delta / 2}$, we get sup | $G_{n}(x)-\Phi(x) \mid=O\left(n^{-\frac{5}{2}}\right)$.

TheOREM 3.2. Suppose that $\sigma_{1}>0, E\left|g\left(X_{1}, \ldots, X_{k}\right)\right|^{3}<\infty$ and

$$
E\left|g\left(X_{j_{1}}, \ldots, X_{j_{k}}\right)\right|^{2}<\infty, \quad 1 \leq j_{1} \leq \cdots \leq j_{k} \leq k .
$$

Then, inequality

$$
\left|P\left(\frac{\sqrt{n}}{k \sigma_{1}}\left(Y_{n}-E Y_{n}\right) \leq x\right)-\Phi(x)\right| \leq \frac{C}{\sqrt{n}\left(1+x^{2}\right)}
$$

holds for all $x \in R$.

Proof. For the first term of the left-hand side of the inequality

$$
\frac{\sqrt{n}}{k \sigma_{1}}\left(Y_{n}-E Y_{n}\right)=\frac{\sqrt{n}}{k \sigma_{1}}\left(U_{n}-\theta\right)+\frac{\sqrt{n}}{k \sigma_{1}}\left(R_{n}-E R_{n}\right),
$$

By Markov's inequality and (3.2) we have for $x \neq 0$

$$
P\left(\frac{\sqrt{n}}{k \sigma_{1}}\left|R_{n}-E R_{n}\right| \geq \frac{C_{1}}{\sqrt{n}}|x|\right) \leq \frac{C_{2}}{n|x|^{2}} .
$$

For $|x| \geq 1$, we have $1+|x|^{2} \leq 2|x|^{2}$ and so

$$
P\left(\frac{\sqrt{n}}{k \sigma_{1}}\left|R_{n}-E R_{n}\right| \geq \frac{C_{1}}{\sqrt{n}}|x|\right) \leq \frac{C_{3}}{n\left(1+|x|^{2}\right)} .
$$

Applying Lemma 2.2, (3.5) and (3.6) to Lemma 2.3, we get the theorem.

TheOREM 3.3. Suppose that $\sigma_{1}>0, E\left|g\left(X_{1}, X_{2}\right)\right|^{3}<\infty$, and $E\left|g\left(X_{1}, X_{1}\right)\right|^{3}<$ $\infty$. Then, the inequality

$$
\left|P\left(\frac{\sqrt{n}}{2 \sigma_{1}}\left(Y_{n}-E Y_{n}\right) \leq x\right)-\Phi(x)\right| \leq \frac{C}{\sqrt{n}(1+|x|)^{3}}
$$

holds for $n \geq 8$ and all $x \in R$. 
Proof. By Markov's inequality and (3.2) we have for $x \neq 0$,

$$
P\left(\frac{\sqrt{n}}{2 \sigma_{1}}\left|R_{n}-E R_{n}\right| \geq \frac{C_{1}}{\sqrt{n}}|x|\right) \leq \frac{C_{2}}{n^{3 / 2}|x|^{3}} .
$$

For $|x| \geq 1$, we have $(1+1 /|x|)^{3} \leq 2^{3} \leq n$ and so

$$
P\left(\frac{\sqrt{n}}{k \sigma_{1}}\left|R_{n}-E R_{n}\right| \geq \frac{C_{1}}{\sqrt{n}}|x|\right) \leq \frac{C_{3}}{\sqrt{n}(1+|x|)^{3}} .
$$

Applying Proposition 2.4, (3.5) and (3.10) to Lemma 2.5, we get (3.9)

Let us consider a bound related with a polynomial. If we allow $n$ to depend on $x$, then we have the following.

TheOREM 3.4. Let $\sigma_{1}>0, E\left|g\left(X_{1}, \ldots, X_{k}\right)\right|^{3}<\infty$ and $E\left|g\left(X_{j 1}, \ldots, X_{j_{k}}\right)\right|^{2}<$ $\infty\left(1 \leq j_{1} \leq \cdots \leq j_{k} \leq k\right)$. In addition, we suppose that $\lim _{|t| \rightarrow \infty}|\eta(t)|<1$. Let $p$ be a polynomial which is positive and increasing over $[0, \infty)$. Then inequality

$$
\left|P\left(\frac{\sqrt{n}}{k \sigma_{1}}\left(Y_{n}-E Y_{n}\right) \leq x\right)-\Phi(x)\right| \leq \frac{C}{\sqrt{n p}(|x|)}, \quad x \in R
$$

holds for a sufficiently large $n$ which depends on $x$.

We can prove this theorem by the similar method to Theorem 2.6, using the BerryEsseen bound of $\left(\sqrt{n} /\left(k \sigma_{1}\right)\right)\left[Y_{n}-E Y_{n}\right]$ (Toda and Yamato, 2001) and its Edgeworth expansion (Yamato et al., 2002). We note that $Y_{n}-\theta$ has a bias but $Y_{n}-E Y_{n}$ has no bias. Under the condition of this proposition we have the Berry-Esseen bound

$$
\sup _{-\infty<x<\infty}\left|P\left(\frac{\sqrt{n}}{k \sigma_{1}}\left(Y_{n}-E Y_{n}\right) \leq x\right)-\Phi(x)\right| \leq \frac{C_{1}}{\sqrt{n}}
$$

and the Edgeworth expansion

$$
\sup _{-\infty<x<\infty}\left|P\left(\frac{\sqrt{n}}{k \sigma_{1}}\left(Y_{n}-E Y_{n}\right) \leq x\right)-Q_{n}(x)\right| \leq \frac{\epsilon_{n}}{\sqrt{n}},
$$

where $\epsilon_{n} \rightarrow 0$ as $n \rightarrow \infty$. We can prove Theorem 3.4 by using these results.

\section{Acknowledgement}

The authors would like to express their thanks to the referee for his careful reading and kind comments. 


\section{References}

Borovskikh, Yu.V. (1996). U-statistics in Banach spaces. VSP, Utrecht.

Koroljuk, V.S. and Borovskich, Yu.V. (1994). Theory of U-statistics, Kluwer Academic Publishers, Dordrecht.

Lee, A. J. (1990). U-statistics, Marcel Dekker, New York.

Mresono, Y. and Yamato, H. (1994). U-statistics and related topics, Sugaku Exposition, 7, 43-58.

Nomachi, T., Kondo, M. and Yamato, H. (2001). Higher order efficiency of linear combinations of U-statistics as estimators of estimable parameters, Scientiae Mathematicae Japonicae, 56, 95-106.

Toda, K. and Yamato, H. (2001). Berry-Esseen bounds for some statistics including LB-statistic and V-statistic, J. Japan Statist. Soc., 31, No. 2, 225-237.

Yamato, H., Nomachi, T. and Toda, K. (2003). Edgeworth expansions of some statistics including LB-statistic and V-statistic, J. Japan Statist. Soc., (to appear).

Zhao, Lincheng (1983). Non-uniform bounds for U-statistics, Chine Annal. Math., Ser. A, 4, No.6, 699-706.

Zhao, Lincheng and Chen, Xiru (1983). Non-uniform convergence rates for distributions of U-statistics, Scientia Sinica, Ser. A, 4, No.6, 699-706.

Received December 12, 2002

Revised April 17, 2003 